\title{
Simulation based Evaluation of a Nonlinear Model Predictive Controller for Friction Stir Welding of Nuclear Waste Canisters
}

\author{
Isak Nielsen, Olof Garpinger and Lars Cederqvist
}

\section{Linköping University Post Print}

\section{Tweet}

N.B.: When citing this work, cite the original article.

Original Publication:

Isak Nielsen, Olof Garpinger and Lars Cederqvist, Simulation based Evaluation of a Nonlinear Model Predictive Controller for Friction Stir Welding of Nuclear Waste Canisters, 2013, European Control Conference (ECC), July 17-19, 2013, Zürich, Switzerland. 20742079.

978-3-952-41734-8/@2013 EUCA

C2013 IEEE. All rights reserved. Personal use of this material is permitted. However, permission to reuse this material for any other purpose must be obtained from the IEEE.

http://ieeexplore.ieee.org/xpl/

Postprint available at: Linköping University Electronic Press http://urn.kb.se/resolve?urn=urn:nbn:se:liu:diva-106112 


\title{
Simulation based Evaluation of a Nonlinear Model Predictive Controller for Friction Stir Welding of Nuclear Waste Canisters
}

\author{
Isak Nielsen $^{1}$, Olof Garpinger ${ }^{2}$ and Lars Cederqvist ${ }^{3}$
}

\begin{abstract}
The Swedish nuclear waste will be stored in copper canisters and kept isolated deep under ground for more than 100,000 years. To ensure reliable sealing of the canisters, friction stir welding is used. To repetitively produce high quality welds, it is vital to use automatic control of the process. This paper introduces a nonlinear model predictive controller for regulating both plunge depth and stir zone temperature, which has not been presented in literature before. Further, a nonlinear process model has been developed and used to evaluate the controller in simulations of the closed loop system. The controller is compared to a decentralized solution, and simulation results indicate that it is possible to achieve higher control performance using the nonlinear model predictive controller.
\end{abstract}

\section{INTRODUCTION}

The Swedish Nuclear Fuel and Waste Management Company (SKB) is responsible for research and development of a long term storage for Sweden's nuclear waste. The proposed solution consists of multiple protective barriers. The spent fuel is first encapsulated in $50 \mathrm{~mm}$ thick copper canisters which are then embedded in bentonite clay about 500 meters below ground in the Swedish bedrock, see Fig. 1.

The repository must keep the spent fuel safe for at least 100,000 years until the radiation has decayed to safe levels. To ensure a sufficiently thick copper corrosion barrier, it is important that the canisters are sealed properly. SKB is currently investigating Friction Stir Welding (FSW) as a method for sealing lids to the $5 \mathrm{~m}$ long and $1 \mathrm{~m}$ diameter canisters. The plunge depth and weld temperature must be controlled within certain limits to produce the corrosion barrier demanded by Swedish authorities. This is currently employed using decentralized control consisting of a cascaded temperature controller, Cederqvist et al. [2], together with a newly proposed plunge depth controller, Nielsen et al. [10], with promising results.

However, cross couplings in the system together with upper and lower bounds on the manipulated variables introduce difficulties with this control design, and the performance gains achieved using a Nonlinear Model Predictive Controller (NMPC) are investigated here.

${ }^{1}$ I. Nielsen is with the Department of Automatic Control, Institute of Technology, Linköping University, SE581 83 Linköping, Sweden isak.nielsen@liu.se

${ }^{2} \mathrm{O}$. Garpinger is with the Department of Automatic Control, Lund University, Box 118, SE-221 00 Lund, Sweden olof.garpingerdcontrol.lth. se

${ }^{3} \mathrm{~L}$. Cederqvist is with the Swedish Nuclear Fuel \& Waste Management Company, Gröndalsgatan 15, SE-572 29, Oskarshamn, Sweden lars.cederqvisteskb.se

\section{THE FRICTION STIR WELDING PROCESS}

The sealing of the canisters is made using FSW, which is a solid state welding method invented in the early 90 's by The Welding Institute (TWI), Thomas et al. [14]. This has become a popular method for forging different metals, such as aluminum, titan, steel and copper.

The basic setup in FSW is seen in Fig. 2, where two copper pieces are welded. The rotating, non-consumable, tool consists of a probe and a shoulder. It generates heat and when the metal is warm enough it is traversed along the joint line, stirring the material from the two pieces into a weld. The heat is a result from friction and plastic deformation of the material.

In contrary to many regular FSW applications where the workpieces are relatively thin and flat, the sealing of the canisters involves thick, cylindrically shaped, metals. A purpose-built welding machine, that copes better with high forces and torques than a standard FSW robot, was installed at the Canister Laboratory in Oskarshamn in 2003.

The tool used in SKB's application consists of a probe that is approximately $50 \mathrm{~mm}$ long, and a convex scroll shoulder with a diameter of $70 \mathrm{~mm}$. The choice of a convex shoulder instead of the commonly used concave shoulder was investigated by Cederqvist et al. [4]. This choice gives smaller variations in plunge depth and spindle torque, which is desirable from a control point of view.

Mayfield et al. [8] conclude that there are three axes in FSW, each with an effort and a flow. Either the effort or the flow is manipulated while the other is determined by the process. In SKB's application, the axial force acting on the tool $\left(F_{z}\right)$, the spindle rotation speed $(\omega)$ and the tool traverse speed $\left(v_{w}\right)$ are possible control signals. These variables are depicted in Fig. 2. The corresponding variables
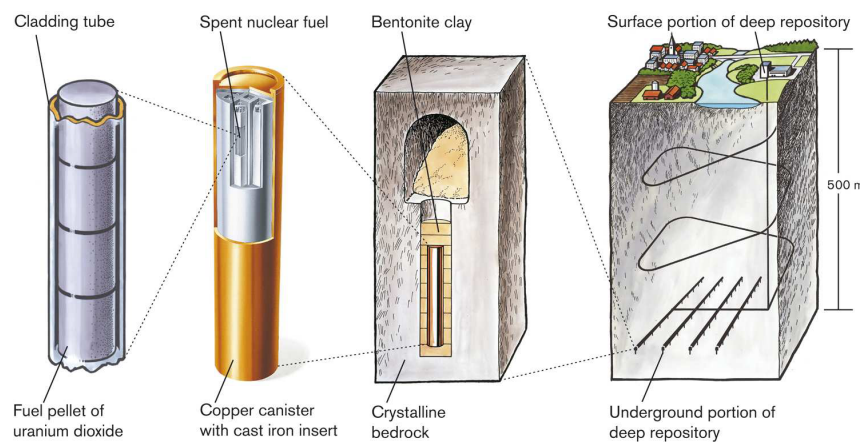

Fig. 1. Multiple protective barriers ensure a safe long term storage of the Swedish nuclear waste. 


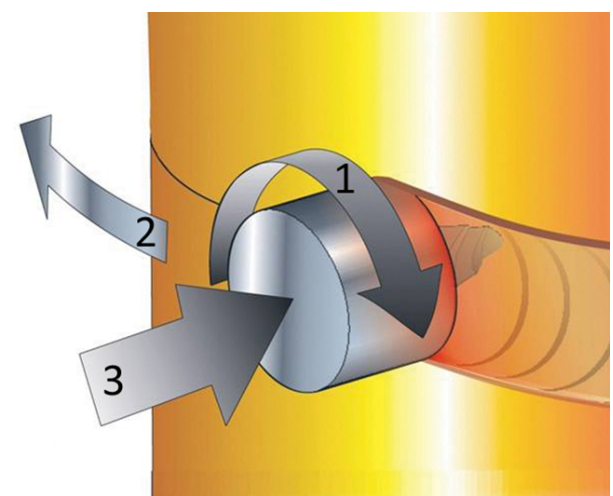

Fig. 2. The manipulated variables are 1) Spindle rotation speed $(\omega)$, 2) Traverse speed $\left(v_{w}\right)$ and 3$)$ Axial force acting on the tool $\left(F_{z}\right)$. The upper part is the lid, and the lower part is the canister.

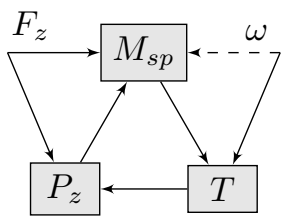

Fig. 3. The relation between the process variables and the manipulated variables in the process. The arrows represent which variables are influencing the others.

plunge depth $\left(P_{z}\right)$, spindle torque $\left(M_{s p}\right)$ and traverse force $\left(F_{t}\right)$ are then given by the process. It was decided to keep the traverse speed constant at $v_{w}=86 \mathrm{~mm} / \mathrm{min}$ and use the other two variables as control signals since their influence on the temperature and plunge depth is greater. The stir zone temperature $(T)$ and power input $(P)$ are also very important process variables. The power input is determined by multiplying spindle torque with spindle rotation speed.

Fig. 3 shows how the process variables are related. The plunge depth affects the spindle torque, which in turn influences the stir zone temperature. The plunge depth depends on the temperature and, hence, there is a triangle of dependences between the process variables. Changing the axial force will affect both plunge depth and spindle torque, while the spindle rotation speed influences the temperature. Observations reveal that the spindle rotation speed influences the torque as well, but this is not accounted for in this article. Similar observations were made by Cui et al. [5].

\section{MODELING OF THE FRICTION STIR WELDING PROCESS}

The combined plunge depth and stir zone temperature control is crucial at the welding sequence start-up, and hence the model is derived for this initial sequence of the weld process. The model relates the two manipulated variables to the controlled variables and is based on a torque model proposed by Schmidt [12]. This torque model is combined with a linearized temperature model and a modified linear plunge depth model.

\section{A. Plunge Depth Modeling}

The plunge depth model proposed in this paper is based on rheology models that are approximate descriptions of viscoelastic materials, and metals at high temperatures can be modeled as such, Flügge [6]. Experimental observations of the SKB data shows that the copper has a tendency to creep, which means that the metal continues to deform even under a constant force.

The tool is considered a rigid body that does not deform at all. Furthermore, it is assumed that the copper closest to the tool have a homogeneous temperature and that the copper outside this region is completely stiff. The strain $\epsilon(t)$ is described by

$$
\epsilon(t)=c \cdot \Delta l(t),
$$

where $\Delta l(t)$ is the deformation of the material and $c$ is a constant. By assuming that the contact area is constant, the stress $\sigma(t)$ can be expressed as

$$
\sigma(t)=\frac{F_{z}(t)}{A},
$$

where $A$ is the contact area and $F_{z}(t)$ is the force acting on the tool. The deformation is the same as the deviation in plunge depth, i.e.

$$
\Delta l(t)=z_{S D}(t)-\bar{z}_{S D},
$$

where $z_{S D}$ is the shoulder depth (plunge depth minus probe length) and $\bar{z}_{S D}$ is the shoulder depth around which the system is linearized.

The model is based on a 3-parameter fluid model, where the idea is to describe the deformation as a combination of two dash-pot elements and one spring element, Flügge [6]. The relation between strain and stress then becomes,

$$
\sigma(t)+p_{1} \dot{\sigma}(t)=\bar{q}_{1} \dot{\epsilon}(t)+\bar{q}_{2} \ddot{\epsilon}(t),
$$

where $p_{1}, \bar{q}_{1}$ and $\bar{q}_{2}$ are constants. Using these equations and the fact that the movement of the tool is subjected to Newton's second law gives

$$
\Delta l(s)=\underbrace{\frac{1}{m p_{1}} \cdot \frac{1+p_{1} s}{s\left(s^{2}+\frac{A q_{2}+m}{m p_{1}} s+\frac{A q_{1}}{m p_{1}}\right)}}_{G_{D}(s)} \Delta F_{z}(s),
$$

as the relation between the change in force and the change in plunge depth. Here, $m$ is the mass of all moving parts and $\Delta F_{z}(s)=F_{z}(t)-\bar{F}_{z}$ is the deviation in axial force from the linearization point $\bar{F}_{z}$.

Observations have shown that the plunge depth is dependent on stir zone temperature as well as the applied axial force. A varying temperature changes the hardness of the heated copper and hence also the parameters considered constant in (5).

This temperature dependence will be included in the final model as an ad hoc solution using a correction factor multiplied with the shoulder depth. The correction factor is assumed affine in the temperature deviation from some temperature $\bar{T}$, and it was estimated using the least-squares 


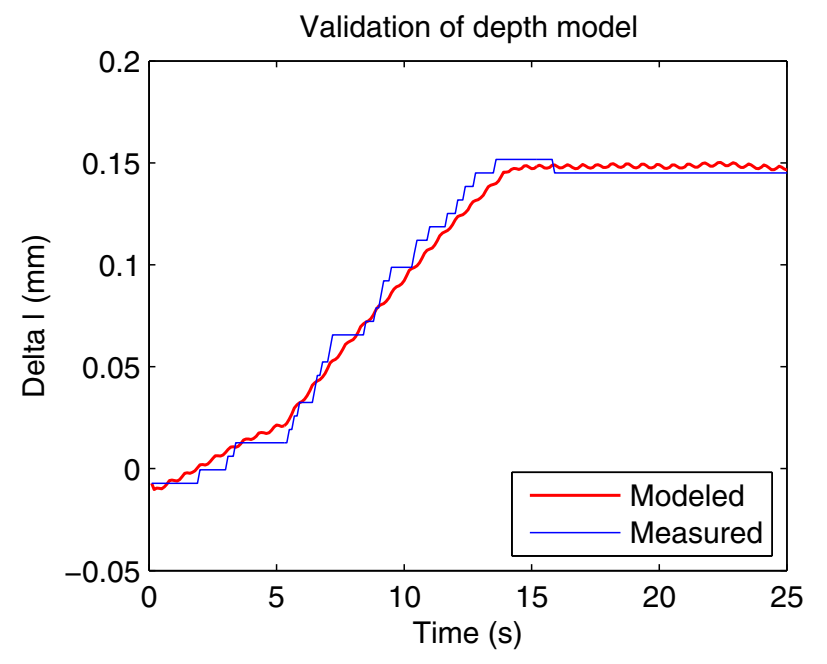

Fig. 4. Validation of the linearized plunge depth model. The model is validated for a step upwards followed by a step downwards in axial force.

method from a data set with constant axial force and varying temperature. The computed correction factor is

$$
C F(\Delta T)=1+0.0021 \cdot \Delta T,
$$

where $\Delta T=T-\bar{T}$ is the deviation in temperature.

Using System IDENTIFICATION TOOLBOX in MATLAB, the linear rheology model combined with the correction factor gives the complete model

$$
\begin{aligned}
\Delta l(s) & =\frac{0.0016 \cdot(1+29.6 s)}{s(1+14.7 s)(1+0.00095 s)} \Delta F_{z}(s) \\
z_{S D}(t) & =\left(\Delta l(t)+\bar{z}_{S D}\right)(1+0.0021 \cdot \Delta T(t)) .
\end{aligned}
$$

The validation of this model is seen in Fig. 4 where a $4 \mathrm{kN}$ step upwards in axial force at 5 seconds is followed by a 4 $\mathrm{kN}$ step downwards at 13.5 seconds.

\section{B. Torque}

The torque sensor measures the motor current and translates it into motor torque $\left(M_{m}\right)$. This must be compensated for in the control system since there is dissipation in the motor and transmission as a result of e.g. friction. Hence, the torque measured by the sensor is a composition of friction torque and spindle torque. The friction torque was measured running the process in air and a friction model is given by

$$
M_{f}(\omega)=243.11-1.305 \cdot \omega+0.001927 \cdot \omega^{2} .
$$

The torque exerted by the tool will be described using a model by Schmidt [12], where the torque is produced in two different ways; slipping and sticking. The slipping, or sliding, condition is when the tool velocity is higher than the material velocity at the interface, resulting in a Coulomb friction between the tool and the copper. When the sticking condition is active, the material is assumed to rotate with the tool and the torque is produced by shear stresses due to plastic deformation of the material. Schmidt et al. [12] argue that it is a combination of these two conditions that together produces the torque. Qian et al. [11] observed that the ratio of torque produced by sticking and slipping conditions varies over a whole weld. In this paper, however, a constant ratio is used.

Introducing the constant state parameter $\delta \in[0,1]$ thus gives

$$
M_{s p}=\delta M_{s t}+(1-\delta) M_{s l}
$$

as the relation for spindle torque. Here $M_{s t}$ is the contribution from the sticking condition and $M_{s l}$ is the contribution from the sliding condition. Looking at an infinitesimal part of the tool, the contact torque $d M_{c}$ is described by

$$
d M_{c}=r \cdot d F=r \cdot \tau_{c} \cdot d A,
$$

where $\tau_{c}$ is the contact shear stress (either $\tau_{s t}$ or $\tau_{s l}$ ), $r$ is the radius and $d A$ is an infinitesimal area section. Integrating this over the tool surface yields

$$
M_{c}=G\left(z_{S D}\right) \cdot \tau_{c}
$$

where the geometric quantity $G\left(z_{S D}\right)$ is dependent on the tool as well as the plunge depth. The sliding shear stress is given by the Coulomb friction $\tau_{s l}(t)=\mu \sigma(t)$, where $\mu$ is the coefficient of friction and $\sigma(t)$ is the contact pressure. The sticking shear stress is assumed to be the same as the copper yield stress, giving $\tau_{s t}=\tau_{\text {yield }}$ where $\tau_{\text {yield }}$ is assumed constant in the relatively narrow band of temperatures used here. The contact pressure $\sigma(t)$ is calculated as force divided by area;

$$
\sigma(t)=\frac{F_{z}(t)}{A\left(z_{S D}(t)\right)},
$$

where $A\left(z_{S D}\right)$ is the depth dependent area given by the tool's projection on the canister surface. Together, these equations give

$$
M_{s p}(t)=\left(\delta \tau_{\text {yield }}+(1-\delta) \mu \sigma(t)\right) \cdot G\left(z_{S D}(t)\right)
$$

as an expression for spindle torque.

In the equations above, the combined inertia of the motor and transmission was not considered. This inertia will act as a low-pass (LP) filter on the measurements. Hence, an LP-filter is added in the motor torque model, giving

$$
\tau_{M} \dot{M}_{m}=-M_{m}+M_{s p}+M_{f},
$$

where $\tau_{M}$ is the time constant of the filter and is estimated from data.

\section{Stir Zone Temperature}

The heating of the stir zone is complex but can be approximated with a linear differential equation relating temperature to power input, see e.g. Cederqvist et al. [3] and Mayfield et al. [8].

Experimental data from the initial sequence of a weld in the lid were used to fit linear process models of varying orders using MATLAB SYSTEM IDENTIFICATION TOOLBOX. The second order linear model with dead time

$$
G_{T}(s)=\frac{18.1}{(1+30.5 s)(1+1.2 s)} e^{-2.8 s},
$$


gave the best fit. The estimation data consisted of step responses in spindle rotation speed while the axial force was held constant.

It is a hypothesis of the authors that the temperature model is actually two models in series; a first order differential equation describing the heating of the copper, and another describing the heating of the tool. The thermo-couple that measures the temperature is placed inside the probe, and therefore the tool temperature is measured, not the stir zone temperature.

\section{Full Model}

The full model consists of the equations for the torque, the extended plunge depth model and the temperature model. The linear models are linearized around points which corresponds to values that are representative when the controller is active. These values are

$$
\begin{array}{cc}
\bar{F}_{z}=78.4 \mathrm{kN}, & \bar{T}=816.4^{\circ} \mathrm{C} \\
\bar{z}_{S D}=2.66 \mathrm{~mm}, & \bar{P}=43.6 \mathrm{~kW}
\end{array}
$$

and the resulting, full model is (omitting time arguments for brevity)

$$
\begin{aligned}
& \Delta T=\frac{18.1}{(1+30.5 s)(1+1.2 s)} e^{-2.8 s} \Delta P \\
& \Delta l=\frac{0.0016 \cdot(1+29.6 s)}{s(1+14.7 s)(1+0.00095 s)} \Delta F_{z} \\
& 0.27 \dot{M}_{m}=-M_{m}+M_{s p}+M_{f} \\
& M_{s p}=(152800+0.435 \cdot \sigma) G\left(z_{S D}\right) \\
& \sigma=\frac{F_{z}}{A\left(z_{S D}\right)} \\
& A\left(z_{S D}\right)=\pi\left(0.015+\frac{20}{3} z_{S D}\right)^{2} \\
& G\left(z_{S D}\right)=2.3 \cdot 10^{-5}+\ldots \\
& \ldots .2 .408 \cdot\left(\left(0.015+\frac{20}{3} z_{S D}\right)^{3}-0.015^{3}\right) \\
& M_{f}=243.11-1.305 \cdot \omega+0.001927 \cdot \omega^{2} \\
& \Delta P=\frac{\left(M-M_{f}\right) \omega \pi}{30000}-43.6 \\
& F_{z}=\Delta F_{z}+78.4 \\
& T=\Delta T+816.4 \\
& z_{S D}=(\Delta l+2.66)(1+0.0021 \cdot \Delta T)
\end{aligned}
$$

which will be used in the controller design. The validation of the model is seen in Fig. 5, where a $4 \mathrm{kN}$ step in axial force is applied at 2 seconds followed by a $-4 \mathrm{kN}$ step at 10.5 seconds. A step of height 60 RPM is made in spindle rotation speed at 19 seconds, followed by two -20 RPM steps at 27.1 and 30.3 seconds. Since the torque measurements are derived from the motor current, there is a peak in the torque after approximately 20 seconds. This peak is a result of the spindle acceleration and is not injected to the process. The coupling between rotation speed and torque is seen after 20 seconds. The measured torque drops, but since the model
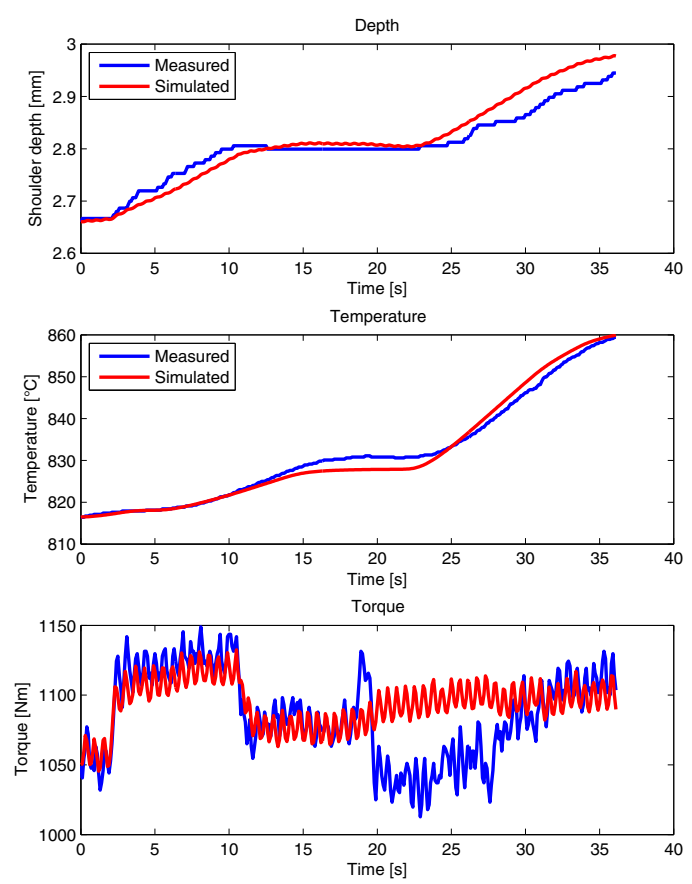

Fig. 5. Validation of the full model. A step in the axial force is applied followed by a step in spindle rotation speed. The model does not describe the relation between spindle rotation speed and torque.

does not capture this relation, the modeled torque is kept at a higher value. It is a hypothesis of the authors that this drop in torque is mainly due to the fact that the motor current is used as an indicator of torque. This conclusion is supported by the modeled temperature which is still good, indicating that the torque drop is a sensor artifact and not due to a drop in spindle torque.

\section{NONLINEAR MODEL PREDICTIVE CONTROL}

The basic idea when using linear or nonlinear MPC is to solve an optimization problem to find the optimal control signal for some performance measure in in every iteration of the control loop. The simulations of the closed loop system are made using the modeling, simulation and optimization software APMONITOR [1]. The continuous model presented in Section III-D is discretized by APMONITOR and the user is not concerned with this matter.

The optimization problem consists of a cost function and a set of constraints that represents the nonlinear model and bounds on the manipulated variables. The cost function is a measure of how well the control performance goals are met, and in this paper the $\mathcal{L}_{1}$ norm is used. This choice is better at explicitly prioritizing the control objectives than the commonly used $\mathcal{L}_{2}$ norm, Hedengren et al. [7]. The idea with this approach is to define a dead-band within which the controlled variable should be kept. If the value is outside this dead-band, it will be penalized by an increased cost. See e.g. Scokaert et al. [13] for a detailed explanation of soft constraints. Slack variables $\left(e_{h i}\right.$ and $\left.e_{l o}\right)$ together with 
inequalities are introduced to relax the use of absolute values. This makes the cost function smooth and continuously differentiable which is a requirement for large-scale Nonlinear Programming (NLP) solvers. Besides the constraints given by the model and limits on the variables, there are also constraints for the upper and lower limits on the dead-band $\left(y_{t, h i}\right.$ and $\left.y_{t, l_{o}}\right)$. The limits will be described by first order linear differential equations with time constants given by $\tau_{t}$. This NLP problem is the same as in Hedengren et al. [7] with some weights equal to zero and no disturbance model, and has the form

$$
\begin{aligned}
& \min _{u} \Phi=\min _{u} w^{T} e_{h i}+w^{T} e_{l o}+(\Delta u)^{T} c_{\Delta u} \\
& \text { s.t. } 0=f(\dot{x}, x, u) \\
& 0=g\left(y_{s}, x, u\right) \\
& a \geq h(x, u) \geq b \\
& \tau_{t} \frac{\partial y_{t, h i}}{\partial t}+y_{t, h i}=s p_{h i} \\
& \tau_{t} \frac{\partial y_{t, l o}}{\partial t}+y_{t, l o}=s p_{l o} \\
& e_{h i} \geq\left(y_{m}-y_{t, h i}\right) \\
& e_{l o} \geq\left(y_{t, l o}-y_{m}\right) .
\end{aligned}
$$

The dynamic model is described by $f(\dot{x}, x, u)$ where $x$ are the states, $u$ the control signal and $\Delta u$ the movement in $u$. The physical interpretation of $x$ is not important since the measurements $y_{s}$ and states are related by $g\left(y_{s}, x, u\right)$. The constraints on the control signals are given by the function $h(x, u)$ together with $a$ and $b$. Further, the set points for the upper and lower limit of the dead band is given by $s p_{h i}$ and $s p_{l o}$. The slack variables measure how far from the desired trajectory the modeled output $y_{m}$ is.

The optimization problem is solved over a finite time horizon which has to be long enough to capture important dynamics. The controller in this article uses the time horizon

$$
t=[0: 0.1: 5,7,10,15,30]^{T},
$$

where MATLAB syntax has been used. The second part of the vector $\left([7,10,15,30]^{T}\right)$ is added to get predictions further ahead without adding a large number of extra variables to the optimization problem.

The most important tuning parameters are the weights used in the cost function, but also the time constant of the response $\left(\tau_{t}\right)$ is a tuning parameter that is set to 5 seconds here. The default solver in APMONITOR is APOPT, and the only changes to the default settings for this solver is the number of nodes used in every prediction horizon which has been set to 2 (default 4). The step sizes in the manipulated variables have been constrained to $1 \mathrm{kN}$ and $3 \mathrm{RPM}$ respectively, preventing excessive movement in the control signals. The dead-bands are set to $\pm 0.1^{\circ} \mathrm{C}$ from the desired stir zone temperature and $\pm 0.002 \mathrm{~mm}$ from the desired plunge depth, and the weights

$$
w=\left[\begin{array}{c}
100 \\
1
\end{array}\right], \quad c_{\Delta u}=\left[\begin{array}{c}
1 \\
0.01
\end{array}\right]
$$

are used. These have been determined in an iterative manner.
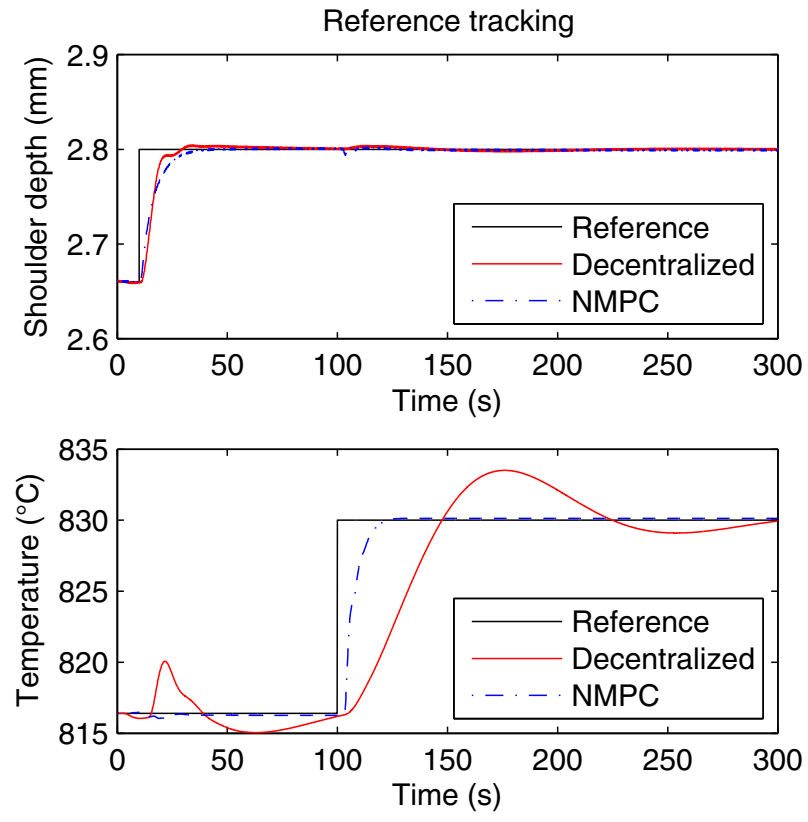

Fig. 6. Simulated controlled variables for the decentralized controller and the NMPC. The NMPC almost completely decoupled closed loop system. The black lines are the references.

\section{SIMULATION RESULTS}

The proposed NMPC scheme has been compared in simulations to the implemented controller proposed in Nielsen et al. [10]. Results both from reference tracking and disturbance accommodation are presented here. Also, model uncertainties has been simulated giving results very similar to the disturbance simulations. These are however not presented here.

\section{A. Reference Tracking}

The closed loop response for steps injected in plunge depth and stir zone temperature are seen in Fig. 6, with corresponding control signals in Fig. 7. The NMPC scheme accomplish an almost decoupled closed loop system, whereas the decentralized controller has some significant interaction between the two controlled variables. The decoupling is achieved by compensating the cross-couplings by an active use of the control signal, see Fig. 7.

\section{B. Disturbance Accommodation}

The closed loop systems have also been simulated for a step disturbance acting on the shoulder depth. This disturbance could be e.g. a sharp edge between the lid and the canister which must be compensated for. The result is seen in Figs. 8 and 9. When the step disturbance is injected, the controllers make a quick drop in axial force, which the NMPC compensates with a quick increase in rotation speed to get a smooth descent. The decentralized controller, on the other hand, does not compensate as well which results in an undershoot. The fluctuations in depth induces changes in stir zone temperature which the decentralized controller cannot compensate for immediately. A similar disturbance enters the temperature at 160 seconds, with a similar behaviour. For 


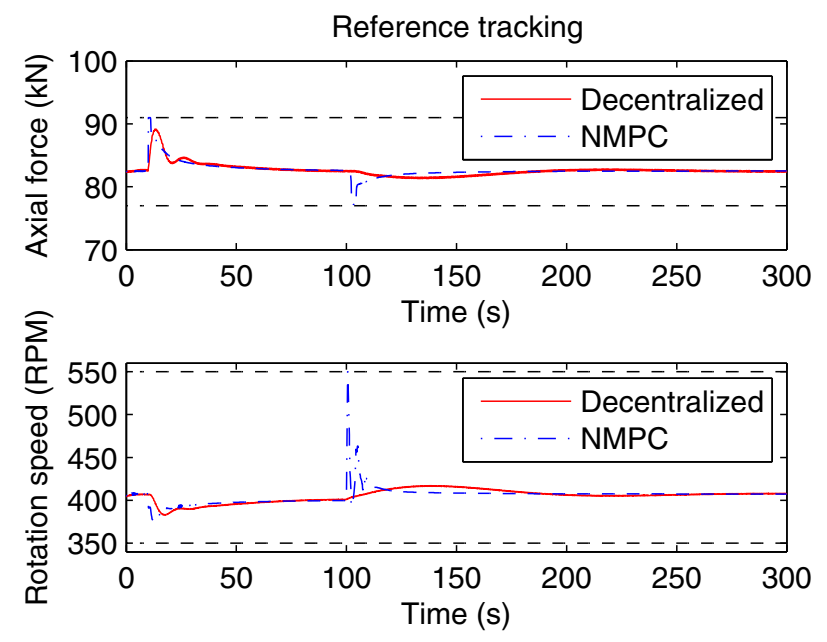

Fig. 7. Simulated control signals for the decentralized controller and the NMPC. The dashed lines are the limits on the manipulated variables.
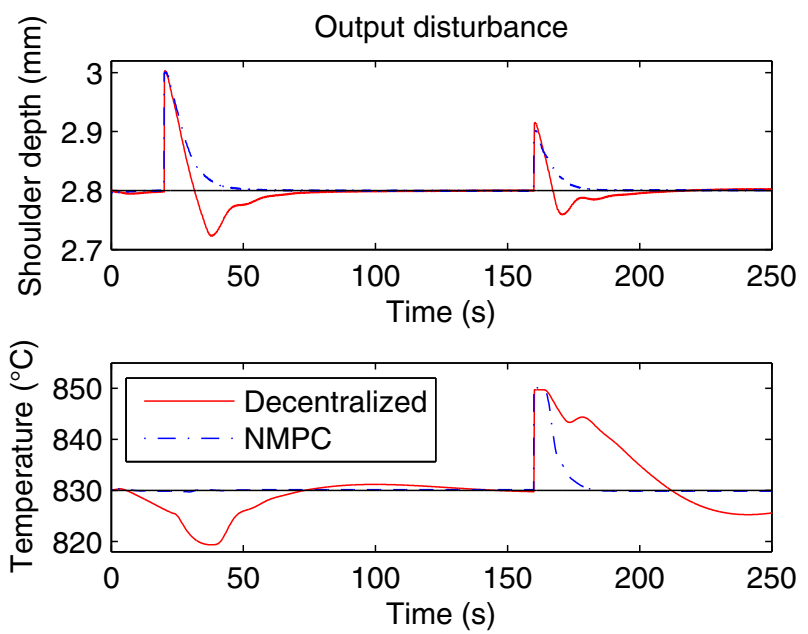

Fig. 8. Simulation of an output disturbance at the plunge depth and the temperature. The NMPC compensates for the disturbances faster than the decentralized controller.

both types of disturbances, the NMPC response is smooth and well behaved, which indicates good robustness towards disturbances. Also, simulations of model uncertainties reveal good robustness towards modeling errors. These are however not included here, since they are very similar to Fig. 8 and 9.

\section{CONCLUSIONS}

This paper introduces the concept of nonlinear model predictive control to FSW applications with promising simulation results. The NMPC manages to almost completely decouple the modeled system with respect to reference tracking, and it handles disturbances well with a fast and damped disturbance accommodation. This is achieved with smooth control signals without any unwanted rapid oscillations. Even though there are strong indications that an NMPC controller gives better control performance than the existing controller, a real time implementation on SKB's friction stir machine is not possible today. The main issue is the limited computational resources in the PLCs used by the

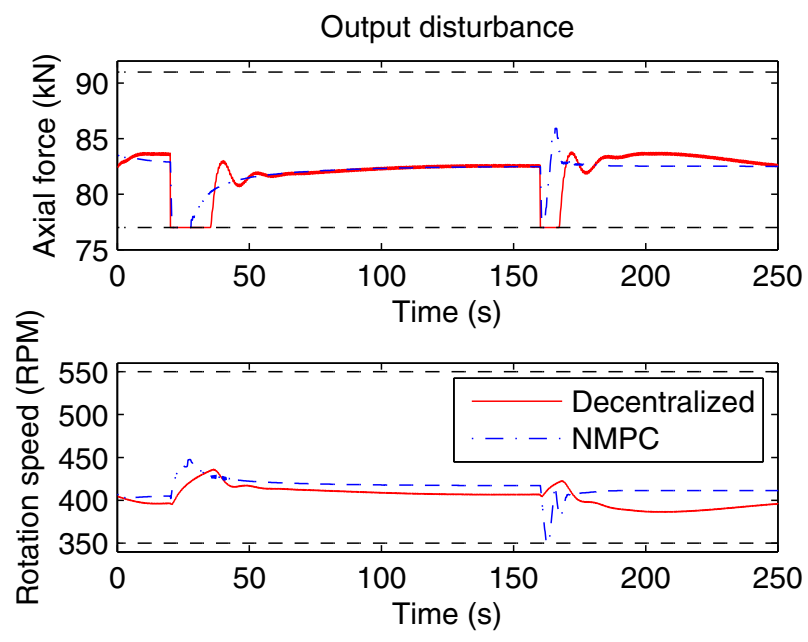

Fig. 9. Simulated control signals when step disturbances entering at the outputs are applied. The NMPC scheme uses rapid changes in the control signals to compensate for the disturbances.

control system. These cannot handle more advanced control algorithms such as the proposed NMPC scheme.

\section{ACKNOWLEDGMENTS}

This research was founded by the Swedish Nuclear Fuel and Waste Management Company.

\section{REFERENCES}

[1] APMonitor. www . apmonitor. com, 2012.

[2] L. Cederqvist, Friction Stir Welding of Copper Canisters Using Power and Temperature Control, PhD. Thesis, Lund University, 2011

[3] L. Cederqvist, O. Garpinger, T. Hägglund, A. Robertsson. Cascade control of friction stir welding process to seal canisters for spent nuclear fuel. Control Engineering Practice, Jan. 2012.

[4] L. Cederqvist, C.D. Sorensen, A.P. Reynolds, T. Örberg. Improved process stability during friction stir welding of $5 \mathrm{~cm}$ thick copper canisters through shoulder geometry and parameter studies. Science and Technology in Welding and Joining 46(2).

[5] S. Cui, Z. W. Chen, J. D. Robson. A model relating tool torque and its associated power and specific energy to rotation and forward speeds during friction stir welding/processing. International Journal of Machine Tools \& Manufacture 50, Sep. 2010.

[6] W. Flügge, Viscoelasticity, Springer-Verlag, 1975.

[7] J. D. Hedengren, R. Asgharzadeh. Tutorial overview of solving $d y-$ namic optimization and advanced control for large scale industrial systems. Preprint to Control Engineering Practice.

[8] D.W. Mayfield C.D. Sorensen. An improved temperature control algorithm for friction stir welding. In 8th International Symposium on Friction Stir Welding, Timmerdorfer Strand, Germany, May 2010.

[9] R.S. Mishra, Z.Y. Ma. Friction stir welding and processing. Materials Science and Engineering R 50, Aug. 2005.

[10] I. Nielsen, O. Garpinger, L. Cederqvist. Decentralized Friction Stir Welding Control on Canisters for Spent Nuclear Fuel. Technical report, http://urn.kb. se/resolve?urn=urn: nbn: se: liu: diva-91571 Linköping University, 2013.

[11] J. W. Qian, J. L. Li, J. T. Xiong, F. S. Zhang, W. Y. Li, X. Lin. Periodic variation of torque and its relations to interfacial sticking and slipping during friction stir welding. Science and Technology of Welding and Joining, Jan. 2012.

[12] H. Schmidt, J. Hattel, J. Wert. An analytical model for the heat generation in friction stir welding. Modelling and Simulation in Material Science and Engineering, Nov. 2003.

[13] P. O. Scokaert and J. B. Rawlings. Feasibility issues in linear model predictive control. A.I.Ch.E. Journal, 45(8), 1999.

[14] Thomas WM, Nicholas ED, Needham JC, Murch MG, Temple-Smith P, Dawes CJ. Friction stir butt welding, Patent PCT/GB92/02203,1991 